\title{
Expected oscillation parameters for red giants from dynamical masses and radii
}

\author{
Nathalie Themeß| ${ }^{1,2,3, \star}$ and Saskia Hekker ${ }^{1,2}$ \\ ${ }^{1}$ Max Planck Institute for Solar System Research, Justus-von-Liebig-Weg 3, 37077 Göttingen, Germany \\ ${ }^{2}$ Stellar Astrophysics Centre, Aarhus University, Ny Munkegade 120, 8000 Aarhus C, Denmark \\ ${ }^{3}$ Institute for Astrophysics, Georg-August Univ. Göttingen, Friedrich-Hund-Platz 1, 37077 Göttingen, Germany
}

\begin{abstract}
Stellar masses and radii of stars with stochastic (solar-like) oscillations can be derived using asteroseismic scaling relations. Here, we predict the asteroseismic observables using the dynamical masses and radii from red-giants in eclipsing binary systems. We show that the predicted frequency of maximum oscillation power $\left(v_{\max }\right)$ is generally lower than the observed one, while the predicted large frequency separation $(\Delta v)$ is in most cases larger than the observed value. This shows that both the scaling relations for $\Delta v$ and $v_{\max }$ used with solar references contribute to the observed differences between dynamical and asteroseismically determined masses and radii.
\end{abstract}

In the era of space missions, in particular Kepler and K2, solar-like oscillations have been observed in a large number of stars. The study of these stochastically excited oscillations provides knowledge about physical properties, such as mean density and surface gravity and thus stellar mass and radius. From the Fourier spectrum of red giants it is possible to directly measure two asteroseismic parameters, the frequency of maximum oscillation power $v_{\max }$ and the large frequency separation $\Delta v$, i.e. the spacing between modes of the same spherical degree and consecutive radial order. These quantities together with the effective temperature $\left(T_{\text {eff }}\right)$ of the star can be used to derive stellar masses $(M)$ and radii $(R)$ through the well-known asteroseismic scaling relations $([1,2])$ :

$$
\frac{\Delta v}{\Delta v_{\odot}} \approx \sqrt{\frac{M / \mathrm{M}_{\odot}}{\left(R / \mathrm{R}_{\odot}\right)^{3}}} \quad \text { and } \quad \frac{v_{\max }}{v_{\max , \odot}} \approx \frac{M / \mathrm{M}_{\odot}}{\left(R / \mathrm{R}_{\odot}\right)^{2} \sqrt{T_{\mathrm{eff}} / \mathrm{T}_{\mathrm{eff}, \odot}}}
$$

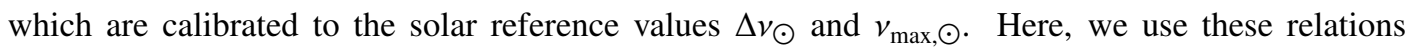
to calculate $\Delta v$ and $v_{\max }$ for known double-lined spectroscopic binaries with red-giant components ([3]). For these systems, dynamical masses and radii can be accurately measured from the binary analysis. We use these measurements in equation 1 and vary the solar reference values as follows: $\Delta v_{\odot}=[134.9,135.0,135.1,135.2] \mu \mathrm{Hz}$ and $v_{\max , \odot}=[3025,3050,3100,3125,3150,3175] \mu \mathrm{Hz}$. This allows us to predict a range of $\Delta v$ and $v_{\max }$ values for each star (see Fig. 1). The ratios between the asteroseismic parameters determined from the scaling relations and those directly measured from the oscillations are shown in Figure 2. We find that the predicted $v_{\max }(\Delta v)$ estimates are lower (higher) than the asteroseismically determined values, with a clear correlation. The right panel of Figure 2 shows $\Delta v$ ratio as function of temperature with the metallicity colour-coded. With this small number of stars we are not able to recover the trend in $T_{\text {eff }}$ and $[\mathrm{Fe} / \mathrm{H}]$ that is present in stellar models ([4]).

\footnotetext{
^ themessl@mps.mpg.de
} 


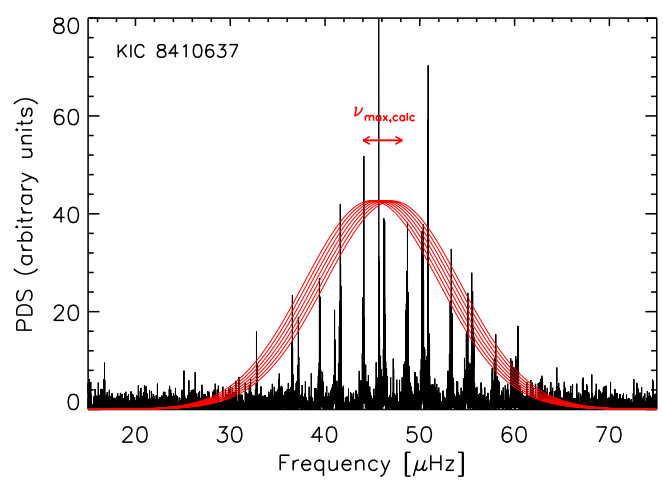

Figure 1. Power density spectrum (in black) of KIC 8410637 centred around $v_{\max }$. The range of predicted $v_{\max }\left(v_{\text {max,calc }}\right)$ values for this red giant is indicated by the red arrow. The corresponding Gaussian fits (multiplied by a factor for visual purposes) are shown in red. No inconsistencies between the predicted and measured values for $v_{\max }$ are apparent.
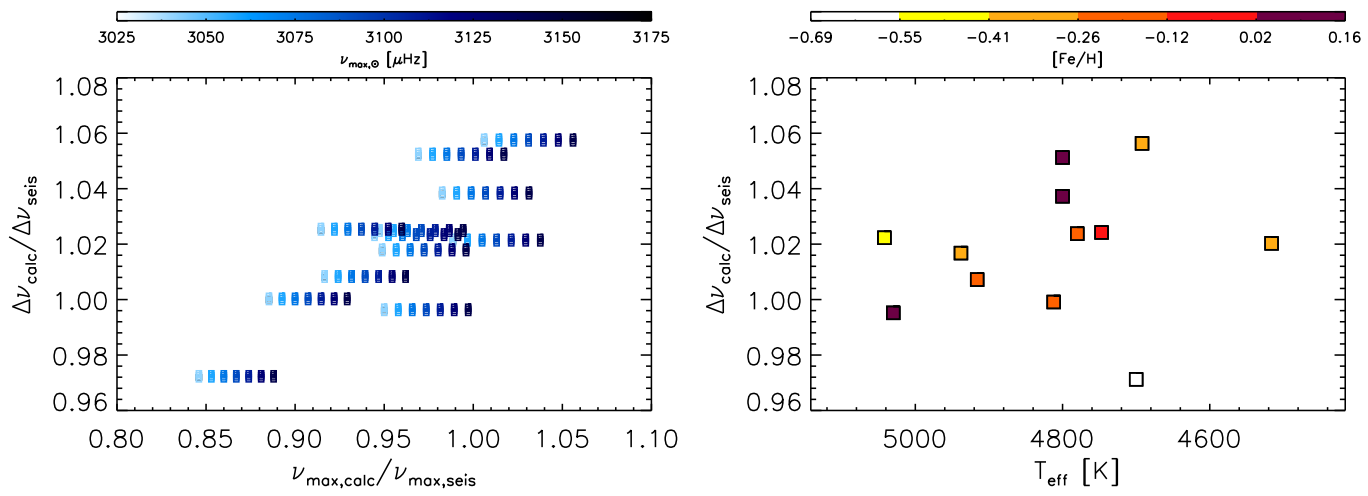

Figure 2. The ratio of predicted $\Delta v\left(\Delta v_{\text {calc }}\right)$ to the seismically determined $\Delta v\left(\Delta v_{\text {seis }}\right)$ as a function of the ratio of predicted $v_{\max }\left(v_{\text {max,calc }}\right)$ over observed $v_{\max }\left(v_{\text {max,seis }}\right)$, left, and as a function of effective temperature $T_{\text {eff }}$, right. The left panel shows all results for the different solar values (see colour bar) and the right panel shows results using a single solar value $\left(\Delta v_{\odot}=135.1 \mu \mathrm{Hz}\right)$ with the points colour-coded for metallicity (see colour bar).

This pilot study tentatively indicates that for red giants not only the $\Delta v$ scaling relation but also the $v_{\max }$ scaling relation suffers from biases in case the solar reference values are used.

Acknowledgments: NT and SH have received funding from the European Research Council under the European Communitys Seventh Framework Programme (FP7/2007-2013) / ERC grant agreement no 338251 (StellarAges). Funding for the Stellar Astrophysics Centre (SAC) is provided by The Danish National Research Foundation (Grant agreement no.: DNRF106).

\section{References}

[1] T.M. Brown, R.L. Gilliland, R.W. Noyes, L.W. Ramsey, ApJ 368, 599 (1991)

[2] H. Kjeldsen, T.R. Bedding, A\&A 293 (1995), astro-ph/9403015

[3] P. Gaulme, J. McKeever, J. Jackiewicz, M.L. Rawls, E. Corsaro, B. Mosser, J. Southworth, S. Mahadevan, C. Bender, R. Deshpande, ApJ 832, 121 (2016), 1609. 06645

[4] E. Guggenberger, S. Hekker, S. Basu, E. Bellinger, MNRAS 460, 4277 (2016), 1606. 01917 